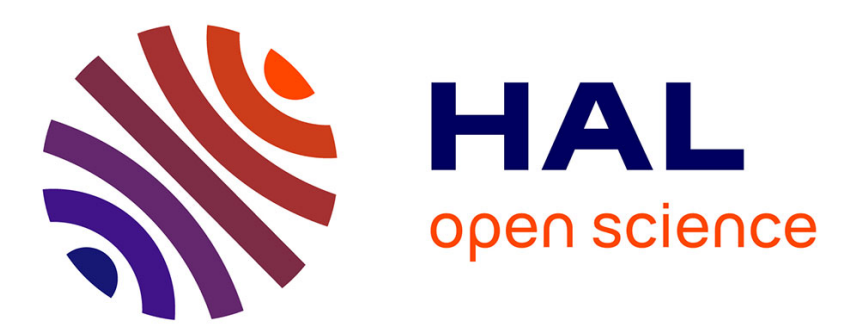

\title{
Entre rituel, mémorial et littérature de propagande. À propos de deux textes ougaritiques au genre mal défini
}

\author{
Jean-Marie Husser
}

\section{To cite this version:}

Jean-Marie Husser. Entre rituel, mémorial et littérature de propagande. À propos de deux textes ougaritiques au genre mal défini. Archimède: archéologie et histoire ancienne, 2020, 7, pp.51-61. halshs-02893143

\section{HAL Id: halshs-02893143 \\ https://shs.hal.science/halshs-02893143}

Submitted on 8 Jul 2020

HAL is a multi-disciplinary open access archive for the deposit and dissemination of scientific research documents, whether they are published or not. The documents may come from teaching and research institutions in France or abroad, or from public or private research centers.
L'archive ouverte pluridisciplinaire HAL, est destinée au dépôt et à la diffusion de documents scientifiques de niveau recherche, publiés ou non, émanant des établissements d'enseignement et de recherche français ou étrangers, des laboratoires publics ou privés. 


\section{ARCHIMĖDE N N 7}

DOSSIER THÉMATIQUE :

GESTES RITUELS. DE LA TRACE À L'INTERPRÉTATION

1 Sylvie DONNAT, Jean-Marie HUSSER

Le rite et ses traces. Perspectives méthodologiques

6 Marie AUGIER

Corps et objets interdits dans les sanctuaires (monde grec, ve av. - III ${ }^{\mathrm{e}}$ ap. J.-C.)

23 Claire CAMBERLEIN

Offrir l'antique à la divinité. L'exemple de l'adyton du sanctuaire de Vryokastro sur l'île de Kythnos (Cyclades)

37 Sylvie DONNAT

Les gestes rituels autour des papyrus-amulettes (Égypte, fin du II millénaire av. n. è.)

51 Jean-Marie HUSSER

Entre rituel, mémorial et littérature de propagande. À propos de deux textes ougaritiques au genre mal défini

62 Anne JACQUEMIN

Rituels efficaces pour fonder ou déplacer un culte en Grèce ancienne

76 Françoise LAROCHE-TRAUNECKER

Les rituels de construction des temples égyptiens. Gestes représentés sur les parois et actes attestés par des fouilles à Karnak

96 Denis MONNERIE

Circulation des objets et élaboration des relations dans les cérémonies du nord de la Kanaky Nouvelle-Calédonie

\section{ACTUALITÉ DE LA RECHERCHE}

294 VARIA 


\title{
ENTRE RITUEL, MÉMORIAL ET LITTÉRATURE DE PROPAGANDE. À PROPOS DE DEUX TEXTES OUGARITIQUES AU GENRE MAL DÉFINI
}

\author{
Jean-Marie HuSSER \\ Professeur émérite d'histoire des religions \\ Université de Strasbourg \\ UMR 7044 Archimède \\ jeanmarie.husser@unistra.fr
}

\section{RÉSUMÉ}

Le corpus des textes ougaritiques comporte deux tablettes qui documentent deux cérémonies spécifiques en lien avec la famille royale (des funérailles, un rituel d'intégration). Or, s'ils contiennent de nombreuses indications rituelles, on ne peut considérer ces textes, écrits en vers, remplis d'éléments lyriques et mythologiques, comme de réels livrets liturgiques, et il est difficile de mesurer leur degré de proximité avec l'action rituelle concernée. Il est développé ici I'hypothèse qu'ils relèvent I'un et I'autre d'un genre littéraire original, dont la fonction était de fixer le souvenir de cérémonies jugées dignes de mémoire concernant la famille royale.

MotS-CLÉS

Ras-Shamra,

Ugarit,

royauté,

rituels,

RS 34.126,

RS 2.002.
Sous la forme d'une évocation poétique, ces textes placent le descriptif du rituel dans le cadre idéologique qui lui donnait sens pour les contemporains.
The corpus of Ugaritic texts includes two tablets which document two specific ceremonies linked to the royal family (a funeral, a rite of passage). Even if they contain numerous hints at ritual, these texts, which are written in verse, and full of lyrical and mythological elements, cannot nevertheless be considered as real liturgical books and it is difficult to measure how close they are to the ritual to which they refer. This paper argues that they belong to a literary genre, whose role it was to anchor in the collective memory ceremonies concerning the royal family which were worthy of remembrance. These texts, in poetic form, locate description of ritual in the ideological context which makes it meaningful for contemporaries.
KEYWORDS

Ras-Shamra, Ugarit, kingship, rituals, RS 34.126 RS 2.002. 
En dehors des nombreux textes rituels bien caractérisés relatifs au culte sacrificiel [1] ou relevant de I'exorcisme, de diverses formes de thérapie et de la nécromancie, le corpus des textes ougaritiques comporte deux tablettes qui documentent chacune une cérémonie en lien avec la famille royale. La première (RS $34.126=$ KTU 1.161) évoque les funérailles de I'avant-dernier roi d'Ugarit, Niqmaddu IV, la seconde (RS $2.002=$ KTU 1.23) se rapporte à une cérémonie dont on a eu jusqu'ici de la peine à préciser la nature et la fonction. Ces deux textes contiennent de nombreuses indications rituelles, mais sont aussi écrits en vers et remplis d'éléments lyriques et mythologiques qui les mettent à part des textes proprement rituels. Il est en conséquence difficile d'établir leur statut et leur lien avec les cérémonies concernées et, de ce fait, de mesurer leur degré de proximité avec l'action rituelle. Pour être plus précis, on s'interroge ici sur la nature des traces du rituel contenues dans ces textes : sontils directement impliqués dans les rites auxquels ils se réfèrent, ou n'en portent-ils qu'un écho plus ou moins lointain ? Et s'ils ne sont que des traces indirectes de ces rituels, laquelle de la pratique ou de l'idéologie des anciens Ougaritains sont-ils le plus susceptibles de documenter?

Ces deux textes sont bien connus des spécialistes, ils ont fait l'objet de nombreuses études épigraphiques et philologiques sur lesquelles je ne vais pas revenir ici. J'ai proposé et argumenté dans un autre contexte une interprétation nouvelle de I'un d'entre eux (KTU 1.23) [2], qui me permet de l'associer à un rite de passage, ou d'intégration, impliquant des princes de rang royal. Cette étude m'a également convaincu des analogies formelles entre ces deux textes qui évoquent tous les deux d'importantes cérémonies impliquant la famille royale. Les pages qui suivent explorent cette piste et tentent de comprendre ce qu'a pu être la fonction de ces textes si particuliers. En plus du style poétique, ils comportent plusieurs éléments formels qui leur sont communs : intervention d'un récitant qui s'adresse aux acteurs du rituel, alternance d'action et de parties lyriques, proximité avec I'institution royale, vœux de « paix » (šlm) adressés à la famille royale.

\section{KTU 1.161}

La tablette RS 34.126 fut retrouvée en 1973 hors de son contexte archéologique initial dans un tas de déblai au sud-ouest de la ville. Malgré un état de conservation médiocre et quelques brisures, elle livre un texte intégralement conservé, écrit sur le recto (25 lignes), la tranche inférieure (2 lignes) et un tiers du verso ( 7 lignes). Sa lecture a été assurée par Pierre Bordreuil et Dennis Pardee [3].

[1] Pour une édition commentée de ces textes, voir PARDEE 2000.

[2] Cette étude a été proposée au colloque « Société et religion à Ougarit », tenu au Collège de France les $15-16$ septembre 2016 ; pour la publication, voir HUSSER 2017.

[3] BordReuil \& PARdeE 1982. 
spr. $d b h . z+l m$

2 qritm . rpi . $a$ [rș]

3 qbitm . qbș.d[dn]

4 qra. ulkn . rp[u]

5 qra . trmn rp[u]

${ }^{6}$ gra. sdn.w.rd[n]

7 qra.tr. Illmn

8 qru. rpim . qdmym

9 qrtim . rpi . arș

10 qbtim . qbs . ddn

11 qra. 'mttmr. mlk

12 gra.u.nqmd. mlk

13 ksi. nqmd . ibky

$14 w . y d m^{\prime} . h d m \cdot p^{\prime} n h$

15 Ipnh. ybky.țhn . mlk

16 w. ybl'. udm'th

17 'dmt. w. 'dmt. 'dmt

18 išhn . špš .

w. išhnn 19 nyr . rbt

'In. špš. tșh

20

$a t r . b^{\prime} l k . l . k s<i>$

atr $21 b^{\prime} l k$. arș. rd

$\operatorname{arss} 22 r d$.

w. špl. 'pr

tht ${ }^{23} s d n$. w. rdn.

tht . tr $^{24}$ 'llmn .

tht . rpim . qdmym

25 tht. 'mțtmr . mlk

26 tht. u. nq[md]. mlk

27 'šty. w.t'ty . tn . ] $w . t^{\prime} y /$

28

$\underline{t} \mid \underline{t} \cdot w \cdot \underline{t} y$.

$\mathrm{a}[\mathrm{rb}]^{\prime} \cdot w \cdot \underline{t}^{\prime} y /$

$29 h m s ̌ \cdot w \cdot t^{\prime} y$.

$\underline{t} t \cdot w \cdot \underline{t}^{\prime} y /$

$30 s ̌ b^{\prime} \cdot w \cdot t^{\prime} y$.

tqdm 'ṣr

31 šlm.

šlm. 'Imr[pi] $w$. šlm . bnh .

šlm . tryl ${ }^{32}$ šlm . bth .

šlm . ugrt ${ }^{33}$ šlm . țgrrh
Livret du banquet des ombres.

Vous avez été appelés, ô Rapa'ūma de la Te[rre], vous avez été convoqués, ô Assemblée de Didanu :

'ULKN le rapa'u fut appelé.

TRMN le rapa'u fut appelé.

SDN-wa-RDN fut appelé.

TR l'éternel fut appelé.

On a appelé les Rapa'ūma antiques,

vous avez été appelés, ô Rapa'ūma de la Terre,

vous avez été convoqués, ô Assemblée de Didanu.

Le roi Ammishtamru fut appelé.

Le roi Niqma[ddu] aussi fut appelé.

Ô trône de Niqmaddu, sois pleuré !

Que l'on sanglote sur l'escabeau de ses pieds !

Que l'on pleure la table du roi (dressée) devant lui !

Et que l'on avale les larmes (versées) pour lui !

Larmes! larmes et larmes encore !

Sois ardente, ô Shapshu,

oui, sois ardente, 19 ô Grand Luminaire !

En-haut, Shapshu, tu crieras :

" À la suite de tes seigneurs, de (ton) trône,

à la suite de 21 tes seigneurs, descends en Terre !

Descends en Terre,

enfonce-toi dans la poussière,

plus bas que SDN-wa-RDN,

plus bas que TR l'éternel,

plus bas que les Rapa'ūma d'autrefois,

plus bas que le roi Ammishtamru,

plus bas aussi le roi Niqmaddu. "

Un (une fois/un jour), on a fait l'offrande $\underline{t}^{\prime} y$,

deux, on a fait l'offrande $t^{\prime} y$,

trois, on a fait l'offrande $\underline{t}^{\prime} y$,

qu[atre], on a fait l'offrande $\underline{t}^{\prime} y$,

cinq, on a fait l'offrande $t^{\prime} y$,

six, on a fait l'offrande $\underline{t}^{\prime} y$,

sept, on a fait l'offrande $\underline{t}^{\prime} y$,

ils ont présenté un oiseau.

Paix,

paix à Ammurapi et paix à ses fils !

Paix à Sharelli, paix à sa maison !

Paix à Ugarit, paix à ses portes ! 
Le texte s'articule en cinq parties de longueurs inégales et suit le déroulement d'un rituel de funérailles, celles d'un souverain dont le nom apparaît à la ligne 13 : Niqmaddu. Après une ligne titre, la première phase de la cérémonie consiste en une « invitation » des ancêtres royaux divinisés, les Rapa'ūma[4] (I. 2-12), suivie d'une lamentation sur le trône, l'escabeau et la table du roi - trois objets symboliques de sa personne et de sa fonction (I. 13-17) [5]. Puis intervient une invocation à Shapshu, divinité psychopompe qui devra conduire le roi défunt dans l'autre monde « à la suite de ses seigneurs », ses prédécesseurs divinisés (I. 18-26), sans doute à l'issue d'une offrande de nourriture renouvelée à sept reprises ou pendant sept jours (I. 27-30). Le texte s'achève avec une formule de bénédiction adressée à la famille régnante et à la cité (I. 31-32).

Cette tablette a en outre la particularité de comporter un titre - ou du moins ce que nous lisons comme tel : spr.dbh. z̧lm. Ce qui pourrait apparaître comme une précieuse indication de son contenu et de sa destination présente pourtant une difficulté propre à désorienter le lecteur. Outre la traduction débattue de $z l m$ [6], c'est le sens précis de spr qui pose ici problème. C'est un dérivé nominal d'une racine pourtant bien connue, mais qui possède un champ sémantique large et développe plusieurs substantifs homographes avec des sens tels que : « écrit, liste, relevé, registre, document (comptable ou administratif), rapport, instruction, missive », en bref, tout type de document écrit. Dans son sens large donc, et en tête d'une tablette inscrite, une

[4] Des nombreux travaux menés sur les rapa'ūma ougaritiques, que I'on retrouve derrière les repha'îm bibliques et phéniciens, il ressort qu'en contexte funéraire, le terme désigne une catégorie particulière de défunts prestigieux, rois ou héros divinisés après leur mort et faisant l'objet d'un culte particulier. Voir ROUILLARD 1999.

[5] Ce sont les trois mêmes éléments du mobilier royal qui sont représentés sur le relief du sarcophage d'Ahiram, une représentation figurée des funérailles royales qui comporte plus qu'une analogie formelle avec ce texte. Cf. infra note 28.

[6] Il y a hésitation entre deux voies d'interprétation du pluriel $z / m$ : soit de $z l$, « ombre, reflet, esprit protecteur », soit de $z / m$, « image, effigie ». Cette seconde interprétation suggérerait que la cérémonie se déroulait en présence de représentations figurées des défunts. Pour un rapide résumé des discussions autour de la traduction de ce texte, voir WYATT 2003, p. 430-441.

[7] Dans CaQuot, TARragon \& Cunchillos 1989, p.105, qui se réfère à l'introduction d'un texte de médecine hippiatrique : $s p r n^{\prime} m s s w m$, «Livret pour la santé des chevaux » (KTU 185.1). Autres traductions proposées : «libretto », « booklet », « ritual ». telle indication aurait tout d'un truisme, d'où les tentatives d'en donner un sens technique plus précis, au risque d'imposer une interprétation a priori sur la fonction de ce texte.

La plupart des traducteurs comprennent spr dans le sens d'un document contenant des instructions pour l'exécution du rituel, d'où la traduction fréquente par « livret», comme A. Caquot : « Livret pour le banquet des ombres »[7], ou plus récemment $\mathrm{N}$. Wyatt : "Order of service for the sacrifice... »[8]. Bien que les termes « livret », « booklet » ou « libretto » ne l'impliquent pas nécessairement, ils suggèrent fréquemment l'idée d'un livret rituel donnant les instructions pour l'exécution d'une cérémonie [9]. Pour d'autres, il s'agirait d'un « rapport écrit », comme l'avaient proposé B. A. Levine et J.-M. de Tarragon [10], ou d'un « document » [11], mais la neutralité voulue de ces termes dissimule en fait I'indécision des traducteurs quant à la nature exacte de ce texte. En gardant la traduction « livret», je I'entends au sens très général d'un document écrit et non pas comme un livret rituel.

$\mathrm{Ce}$ texte évoque les funérailles du roi Niqmaddu [12] et, tel qu'on le comprend, il décrit une cérémonie destinée à permettre au roi défunt de rejoindre la communauté des ancêtres dynastiques. Il commence avec l'invitation faite à ces derniers, les rapa'ūma, de participer au repas funéraire $(d b h \mathrm{z} / \mathrm{m})$ qui leur est offert à cette occasion, peutêtre pendant sept jours - durée probable des funérailles. À l'issue de quoi, ces mânes ancestraux sont invités, sous la conduite de la déesse Shapash, à

[8] WYATT 1998, p. 431, propose comme parallèle l'expression hébraïque zôt tôrat zebah $(\mathrm{Nb} 7,11)$ qui introduit un texte prescriptif du rite sacrificiel. Mais si l'hébreu tôrah signifie bien « instruction », avec l'idée d'un savoir traditionnel, ce n'est pas le cas de $s p r$, dont le champ sémantique est moins précis en dehors du fait qu'il s'agit d'un écrit.

[9] « This text is probably the libretto of the great funeral celebration of the descent of the kings of Ugarit to the « underworld » or simply the anniversary of their death » (Del Otmo 1999, p. 193). «Dans ce texte nous avons le livret, en forme poétique, du rite d'ensevelissement d'un roi d'Ougarit » (PARDEE 2000, vol. 2, p. 824).

[10] LEVINE \& TARRAGoN 1984, p. 649 « written record »; SPRONK 1986, p. 189, et DE MOOR 1987, p. 166 : « report».

[11] PARDEE 2002, p. 87 : «Document of the sacrificial liturgy of the Shades ».

[12] Il s'agit de Niqmaddu IV, décédé en ca 1210, le père d'Ammurapi II, dernier roi d'Ugarit. Cette identification est rendue possible par la mention, dans la bénédiction finale (ligne 31), de Sharelli à côté d'Ammurapi, laquelle était la veuve de Niqmaddu IV et la mère d'Ammurapi. Elle assura la régence pendant une dizaine d'années et resta par la suite associée au règne de son fils. Voir FREU 2006, p. 137-164. 
emporter avec eux le roi défunt dans le monde souterrain. On imagine donc, à l'occasion de cette cérémonie, un double cortège : le cortège funèbre transportant la dépouille du défunt jusqu'à son tombeau, et le cortège des ancêtres invités à la cérémonie. À leur point de rencontre le «banquet des ombres » tient lieu de réunion familiale où morts et vivants communient au même repas avant de s'en retourner chacun dans son monde respectif. En repartant, les rapa'ūma, emporteront avec eux le défunt. Cette interprétation du texte est la plus généralement admise ; je ne souscris pas aux hypothèses - à mes yeux peu convaincantes - de D. Tsumura et d'autres[13] qui voient dans atr (lignes 20-21) un substantif désignant un «lieu », ou un édifice, dont la nature réelle ou symbolique n'a jamais pu être clairement définie. Mais cette discussion a peu d'importance ici.

Ce texte remarquable est le seul exemple de texte funéraire livré par le site de Ras Shamra, et le déroulé harmonieux de la cérémonie qu'il présente invite naturellement à y voir un livret liturgique destiné à guider l'accomplissement des funérailles royales. Il est cependant peu probable qu'il ait rempli une telle fonction, car plusieurs de ses particularités empêchent de le considérer comme un texte rituel :

$1^{\circ} \mathrm{Si}$ le texte suit effectivement le déroulement d'une cérémonie funéraire, sa brièveté lui enlève toute pertinence pratique pour accompagner et guider dans le détail un tel rituel qui devait se dérouler sur un temps que I'on peut supposer assez long, peut-être plusieurs jours, si l'énumération des sept offrandes doit s'entendre comme une réitération du rituel pendant sept jours.

$2^{\circ}$ À l'appui de cette observation, la liste des ancêtres royaux invoqués aux lignes $2-12$, les rapu'ūma, apparaît nettement comme un abrégé d'une liste beaucoup plus longue, comme en atteste le texte RS 24.257 (KTU 1.113) qui nous a transmis une liste des ancêtres royaux divinisés. Un véritable texte rituel n'aurait certainement pas abrégé une telle litanie des ancêtres convoqués à la fête s'il s'agit de n'oublier personne. Cette liste donne d'ailleurs un abrégé qui se veut représentatif de l'ensemble, en englobant d'abord tous les ancêtres dans un collectif général (rpi.arș // qbș.ddn), puis à travers quatre noms propres (sans doute significatifs, mais inconnus de nous), puis dans un nouveau collectif qui semble désigner les plus anciens d'entre eux (rpim.qdmym), et enfin en nommant deux des plus récents, Ammishtamru et Niqmaddu.

$3^{\circ} \mathrm{Il}$ est vraisemblable que les funérailles royales suivaient un rituel qui ne variait guère d'un souverain à l'autre et dont la mémoire était bien établie par la tradition. Si on replace ce texte dans la chronologie générale d'Ugarit, Niqmaddu IV étant décédé en ca 1210, cela situe sa composition dans les vingt-cinq dernières années de la ville [14], et on peut supposer que l'auteur évoque une cérémonie à laquelle il a personnellement assisté. Cette référence à une cérémonie spécifique, très proche de I'auteur et des destinataires du texte, ne permet pas de considérer celui-ci comme le livret rituel servant de guide liturgique pour les funérailles royales en général. Il apparaît plutôt comme un écrit circonstanciel relatif à ces funérailles particulières.

$4^{\circ}$ Enfin, la forme poétique de l'ensemble est étrangère aux textes rituels prescriptifs, toujours rédigés en prose. Dennis Pardee a tenté d'expliquer cette particularité par le fait que le texte fait intervenir des divinités dans l'action rituelle (les rapa'ūma et Shapash), à la différence des textes rituels en prose qui règlent les sacrifices accomplis par des acteurs humains [15]. Cette explication n'est pas convaincante car, en attirant très justement l'attention sur la participation d'acteurs divins à l'action liturgique, Pardee souligne en même temps ce qui rapproche ce texte de la littérature épique et le distingue assez radicalement des textes de la pratique. Je reviens plus loin sur ce point.

S'il ne s'agit pas d'un livret rituel, quelle pouvait être alors la finalité de ce texte singulier ? La comparaison formelle avec le texte RS 2.002 (KTU 1.23), on va le voir, enlève un peu de cette singularité en rapprochant ces deux textes dans leur forme et, sans doute, dans leur fonction et leur rapport au rituel.
[13] TSUMURA 1993, repris, entre autres, par SURIANO 2009.

[14] Rappelons que l'incendie et la ruine définitive d'Ugarit, attribués à un raid des Peuples de la Mer, se situent vers 1185 . Pendant ce dernier quart de siècle, la vie d'Ugarit fut prospère et relativement paisible, sous Niqmaddu IV (ca 1220-1210), sous la régence de sa veuve Sharelli (ca 1210-1200), en dépit d'une première alerte sous la menace des Šikila, puis durant une première partie du règne personnel de son fils Ammurapi II.
La situation se détériora rapidement et inexorablement durant les dernières années de ce dernier, jusqu'à la destruction définitive de la cité. Sur ce contexte historique des derniers règnes, voir FREU 2006.

[15] « The poetic form of the text appears, therefore, to reflect the perception at Ugarit that talk about gods was to be poetic in form (as opposed to the essentially administrative talk about the care and the feeding of the gods in their earthly sanctuaries that characterizes the prose ritual texts) 》(PARDEE 2002, p. 86). 


\section{KTU 1.23}

La tablette RS 2.002 (KTU 1.23) fut retrouvée en 1930 dans la maison du Grand prêtre [16]. Il lui manque un morceau à l'angle supérieur droit et la surface est abîmée sur la partie droite du recto ; il en résulte de nombreuses lacunes au début et à la fin du texte qui en rendent I'interprétation difficile, mais l'écriture est par ailleurs soignée et bien lisible. Le texte est aéré et divisé en petites unités par des traits horizontaux au recto, avec vingt-neuf lignes écrites et neuf traits de séparation. Il est en revanche beaucoup plus serré au verso, où les quarante-et-une lignes de texte ne laissent plus aucune place pour d'éventuels traits de séparation, indice, sans doute, d'une mauvaise gestion de l'espace de la part du scribe. Le texte semble néanmoins intégralement contenu sur la tablette, car les dernières lignes du verso (incomplètes) sont à nouveau un peu plus aérées et s'interrompent avant la tranche inférieure. Il est trop long pour être reproduit ici intégralement, aussi je n'en donne que les vingt-neuf premières lignes qui occupent tout le recto de la tablette et comportent l'essentiel des indications rituelles [17].

Sur cette face de la tablette, des traits horizontaux répartissent le texte en petites unités qui mêlent des récitatifs rythmés, des morceaux lyriques et des indications rituelles. La partie mythologique proprement dite commence à la ligne 30, sur la tranche inférieure de la tablette, et occupe le verso. Il y est question de I'union d'El avec deux femmes et de deux naissances gémellaires qui s'ensuivent, celles des divinités Shahar et Shalim d'une part, et celles des énigmatiques 'ilūma na'mūma d'autre part. Cette désignation, habituellement traduite «les dieux gracieux » en français [18], ne nous dit rien de l'identité de ces personnages que nous devons déduire d'une compréhension globale du texte. Les références au rituel apparaissent dans la première partie du texte (lignes 1-29), tandis que le récit de la hiérogamie avec les deux femmes et de la double naissance occupe le centre du texte (lignes 30-67). Dans les dernières lignes (68-76) s'opère un retour des références à l'action rituelle sur un mode narratif et le mythe fusionne avec celle-ci.

[16] Editio princeps dans VirolleAu 1933.

[17] Pour les discussions et le commentaire philologiques, qui ne concernent pas directement mon propos, je renvoie à la traduction annotée de Nicola Wyatt (WYATT 1998, p. 430-441) ainsi qu'à la récente étude de Mark Smith (SMITH 2006). La traduction ici proposée est la mienne.

[18] Ou « Goodly Gods » en anglais, pour reprendre I'expression de SMITH 2006.

19] Restitution à partir des lignes 58 et 61 .

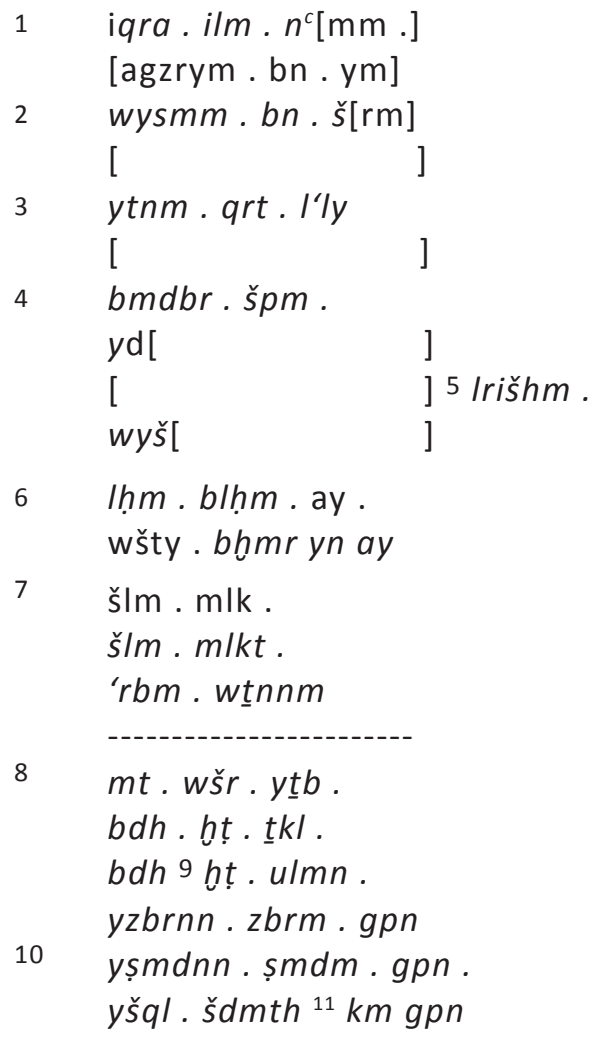

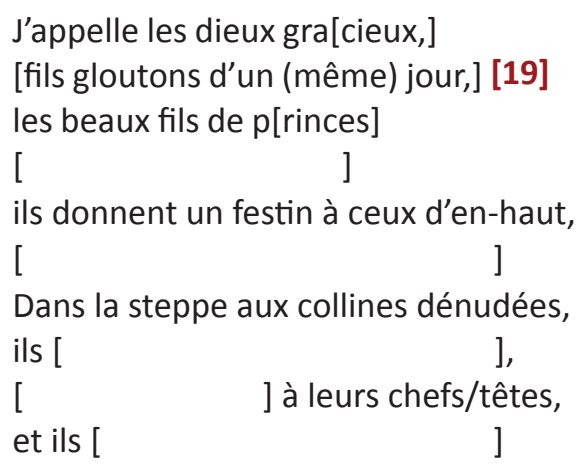

Allez, nourrissez-vous de pain !

Allez, buvez aux cuves de vin!

Paix au roi !

Paix à la reine,

aux entrants et aux archers !

L'époux princier est assis ;

dans sa main, un sceptre stérile, dans sa main, un sceptre veuf. L'émondeur va l'émonder (comme) un cep, le lieur va le lier (comme) un cep, il fera choir son sarment comme un cep! 
šb'd. yrgm . 'I.'d. w'rbm . t'nyn

w. šd. šd. ilm .

$\check{s} d$. atrt . wrhm $m<y>$

'l . išt. šb'd.

ǵzrm g.țb.

gd. $b h \mathrm{l} / \mathrm{b}$

annh bhmat

$w^{\prime}$ l. $a g n$. šb'dm .

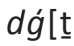

tlkm . rḥmy . wtṣd

[ ] 17 thgrn.

ǵzr.n'm.[ ]

$w s ̌ m . ' r b m \cdot y r[g m]$

[

$m \underline{t} b t . i l m . \underline{t} m n$. $\underline{t}[\mathrm{mn}]$

[

pamt. šb' [

iqnu. šmt [

[

thn . šrm . [

iqran . ilm . $n^{\prime} m m$

[agzr ym . bn .]ym

ynqm . bap zd. atrt .

[ ]

špš . mṣprt . dlthm

[ ] 26 wǵnbm.

šlm . 'rbm . thnnm

$h / \mathrm{km}$. bdbh n'mt

šd. ilm .

šd . ațrt. wrhmy

$\mathrm{il}[\mathrm{m}] \cdot \mathrm{yt} b$
Sept fois on récite, sur le podium, et les entrants font le répons :

La campagne est campagne des dieux, campagne d'Ashirat et de Rahamay.

Sur le feu sept fois,

les jeunes gens à la belle voix,

de la coriandre dans le lait,

de la menthe dans le caillé,

et sur le chaudron encore sept fois, l'enc[ens

Rahamay s'en va chasser,

[ ] elle s'est ceinte,

un gracieux jeune homme [

Et le nom des entrants on ré[cite]

Les sièges des dieux huit par $\mathrm{h}$ [uit]

sept fois [

Lapis-lazuli, cornaline

Les princes/chantres répètent [

Je veux appeler les dieux gracieux, [fils gloutons d'un (même)] jour, qui tètent les seins d'Ashirat,

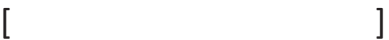

Shapash est celle qui prend soin de leur faiblesse

[ ] et des grains de raisin.

Paix aux entrants, aux archers,

qui processionnent lors du sacrifice de perfection [20].

« Campagne des dieux,

campagne d'Ashirat et de Rahamay. "

Les dieux ont pris place.

Le texte se poursuit avec l'évocation de la hiérogamie d'El et de la naissance des « dieux gracieux » qui, après sept années passées à vivre de chasse «parmi les pierres et les arbres », " entrent » dans l'espace des cultures pour se nourrir de pain et de vin.

20] Ce type de sacrifice, $d b h n^{\prime} m n t$, n'est attesté dans aucune des listes sacrificielles du corpus ougaritique. Je considère que ce prédicat $n^{\prime} m n t$ réfère à cette cérémonie qui célèbre les « dieux» $n^{\prime} m m$, et que ces dérivés de la racine $n^{\prime} m$ signifient la qualité éminente de ces enfants royaux « gracieux, nobles, accomplis » et parvenus à la « perfection » de leur âge. Pour la discussion à ce sujet, voir HUSSER 2017, p. 689-690. 
Les indications rituelles précises [21] se retrouvent presqu'exclusivement dans cette première partie, aux lignes $12 ; 14-15 ; 18-22$ (dont la moitié seulement est préservée) ; $29 ; 57$, soit dix lignes sur soixante-seize. En outre, le récitatif évoque divers rituels sur le mode narratif : un festin rituel (I. 1-6), un rite de circoncision prénuptiale (I. 8-10), une procession des «entrants » (I. 27). En dépit de ces éléments de rituel, il est difficile de voir dans ce texte un livret liturgique destiné à guider l'action des officiants, car ces indications sont brèves et ne constituent pas une séquence suivie de gestes.

En revanche, le rapport entre mythe et rite paraît essentiel dans ce texte qui associe une cérémonie à un récit mythologique, et on doit considérer que ce dernier constituait le référent idéologique à l'action rituelle. Par sa disposition générale et par son contenu cependant ce texte diffère des textes dits « para-mythologiques »[22], ainsi désignés parce qu'ils associent un bref récit mythique à une recette rituelle ou thérapeutique. Ici, le mythe est davantage évoqué que véritablement raconté, le récitatif est allusif et tissé de métaphores, plus lyrique que narratif. Quant aux fragments rituels, ils renvoient à une cérémonie publique complexe impliquant de nombreux acteurs, alors que les textes para-mythologiques concernent des pratiques privées. Enfin, comme KTU 1.161 précédemment étudié, le texte est entièrement à la forme poétique, aussi bien les références rituelles que le récitatif mythologique, et sa disposition graphique présente la particularité rare de respecter assez généralement la stichométrie dans les retours à la ligne.

Sur le fond, et sans entrer dans la discussion détaillée de son interprétation, on remarque que le texte est structuré au moyen de quelques oppositions sémantiques significatives. Ainsi, šedu, la campagne (I. 28 ; $68)$, et madbaru, le territoire sauvage, espace ouvert de la chasse $(I .4 ; 64 ; 68)$, s'opposent à mudara 'u, I'espace des cultures et des semailles (I. 68-69), enclos d'un mur (I. 70-71). De même, oiseaux et poissons, nourriture sauvage (et crue ?) des 'ilūma na'mūma (I. 62-64), s'opposent au pain et au vin, la nourriture civilisée du banquet $(1.6 ; 71-75)$ auquel ils sont conviés. De plus, ces oppositions sont disposées de façon à insérer l'ensemble du texte dans une série $d^{\prime}$ inclusions qui associent étroitement la première partie (le rituel, I. 1-29) à la fin de la seconde partie où le mythe fusionne dans le rituel (I. 65-76). Une première inclusion est constituée par l'invitation à un festin au début du texte (I. 6), qui se réalise à la fin (I. 71-74). En plus du festin, le texte très abîmé des lignes 2-5, qui semble donner en préambule une présentation générale de l'action qui va suivre, mentionne la « steppe aux collines dénudée » ( $m d b r$ špm) qui annonce le monde sauvage décrit aux lignes 65-68, lieu où résident les 'ilūma na'mūma pendant sept années, avant d'intégrer le territoire cultivé (I. 68-71). Enfin, le texte joue sur une troisième inclusion, construite avec la fréquente utilisation de la racine 'rb, « entrer», qu'on voit notamment sous la forme d'un participe pluriel ('rbm) à plusieurs reprises dans la première partie du texte où sont concentrées les références au rituel $(1.7 ; 12 ; 18 ; 26)$. Ces 'āribūma sont des acteurs essentiels de la cérémonie, des « entrants » dont la fonction rituelle semble signifiée par le titre qu'ils portent. Ils font I'objet d'une double bénédiction en compagnie du couple royal $(I .7 ; 26)$, et interviennent encore à deux reprises (I. 12 et 18 ). Or, à la fin du texte, après le mythe de la hiérogamie et de leur naissance, le récitatif évoque les « dieux gracieux » qui « entrent » ( $\left.w^{\prime} r b \mathrm{hm}\right)$ dans le territoire des cultures (I. 69-71), après avoir erré et chassé pendant sept années « aux confins de la steppe » (tșdn pat $m d b r$, I. 67-68).

Dans la perspective holiste qui est celle de toute action rituelle dans les sociétés antiques et traditionnelles, le rite met en relation des êtres aux statuts variés et aux fonctions différentes, des acteurs visibles et d'autres invisibles, abolissant les barrières qui les séparent ordinairement. Autrement dit, les acteurs du mythe sont aussi des acteurs du rituel, par la puissance performative du verbe et la dimension symbolique du geste. Ainsi, les 'ilūma na'mūma, « dieux gracieux », personnages centraux, interviennent autant dans le rituel que dans le mythe référent, et leur identité est la clé de compréhension de ce texte.

Dans l'interprétation que je propose de ce texte [23], le mythe évoqué - la double hiérogamie $\mathrm{d}^{\prime} \mathrm{El}$ et la double naissance de jumeaux divins - sert de modèle mythique et de référent idéologique au

[21] Ces indications, pour « précises » qu'elles soient, n'en sont pas pour autant intelligibles pour nous ; elles gardent un caractère allusif du fait de leur extrême concision.

[22] Le qualificatif « para-mythologique » s'applique à un groupe de neuf textes dont Dennis Pardee a défini les caractéristiques communes. D'après leur lieu de

découverte et les documents qui leur étaient associés, ces textes faisaient partie de « la bibliothèque d'un prêtre du culte officiel d'Ougarit en même temps au service d'une certaine clientèle, peut-être même la famille royale... » (PARDEE 1988, p. 262).

[23] HUSSER 2017. 
mariage du roi, à la conception et à la naissance des princes royaux, ici désignés comme des « dieux gracieux ». Dans l'idéologie royale, les fils du roi sont effectivement divins, considérés comme « fils d'El » et nourris aux seins de la déesse Athirat dans l'imaginaire mythique, d'où leur désignation ici comme 'ilūma na'mūma. Quant au rituel auquel renvoie I'ensemble du texte, il s'agit selon moi d'une cérémonie d'intégration de ces princes royaux, « dieux gracieux », dans la société des jeunes garçons de la noblesse. En raison de leur rôle dans cette cérémonie, ils sont donc également appelés 'āribūma, «les entrants », dans le texte qui s'achève avec le récit de leur « entrée » dans l'espace civilisé. Le contexte serait donc celui d'un rite de passage au sortir de la petite enfance, après une période de sept années passées symboliquement dans le monde sauvage, c'est-à-dire en marge des usages de la société des jeunes gens et des adultes. En plus d'indices textuels précis, cette hypothèse s'appuie sur un ensemble de recherches récentes qui concluent à l'existence de rites de passage dans les sociétés du Bronze au Proche-Orient ancien, et à Ugarit notamment [24].

De mon point de vue donc, et comme pour KTU 1.161 , nous n'avons pas affaire à un texte rituel, en dépit de son étroite relation avec une cérémonie. Sa structure très ferme rapidement décrite ici en fait un texte à caractère littéraire, proche du style épique, qui suit une ligne narrative claire malgré les nombreuses interventions du rituel. La forme poétique utilisée dans ces deux textes pose donc la question de leur genre littéraire, de leur fonction et de la nature de leur relation avec les rituels concernés.

L'usage de la forme poétique dans les textes rituels (phénomène rare) a été brièvement étudiée par Dennis Pardee qui distingue trois catégories : «(1) poetic rituals ; $(2)$ poetic texts with ritual overtones of one kind or another ; (3) prose ritual texts with embedded poetry »[25]. À la première catégorie, celle des rituels poétiques (poetic rituals), Pardee associe uniquement le texte des funérailles royales que nous avons vu plus haut, KTU 1.161 (« the only clear example »); dans la catégorie 2 (poetic texts with ritual overtones), il compte certains textes para-mythologiques, ainsi que les textes KTU 1.23 (celui qui nous occupe) et 1.169 (une incantation contre l'impuissance masculine), très différents l'un de l'autre pour le contenu ; pour la catégorie 3, il cite KTU 1.119, qui introduit une prière à Baal en vers (lignes 26-36) à la suite d'une liste sacrificielle en prose.

Sans surprise, il apparaît de cette typologie et d'un décompte des textes para-mythologiques, que les seuls textes poétiques à caractère incontestablement rituel sont des prières (KTU 1.119 : 26-36), des morceaux hymniques (KTU 1.113) ou des incantations (KTU $1.100 ; 1.107 ; 1.169$ ). Autrement dit, la forme poétique n'est utilisée que pour des textes faisant l'objet d'une récitation dans le cadre d'un rituel, mais jamais pour des textes prescriptifs des gestes du rituel. Or, nos deux textes ne sont ni des hymnes ni des prières à réciter pendant une cérémonie, même s'ils contiennent effectivement quelques fragments hymniques ou mythologiques qui devaient accompagner les cérémonies évoquées. En conséquence, la forme poétique qui caractérise KTU 1.23 et 1.161 suggère pour ces textes une autre fonction que des prescriptions ou des aide-mémoires pour les rituels concernés [26].

Le caractère allusif des indications rituelles n'entre pas en considération ici ; comme c'est généralement le cas dans notre documentation ancienne, le détail des cérémonies auxquelles ces textes réfèrent était bien connu de leurs destinataires, ce qui dispensait de tout préciser dans l'écrit. Mais si ces deux textes ne livrent des rituels qu'une trame générale indiquée par de brèves indications et des citations fragmentaires, tout cela est enrobé dans un tissu de références à I'univers mythologique qui semble bien constituer le cœur de leur message. Conséquence de cette facture hybride : aux yeux des observateurs extérieurs que nous sommes, ces textes sont trop imprécis pour établir la matérialité des rituels et le détail de leur déroulement. Ils sont en revanche d'excellents témoignages sur la signification de ces cérémonies pour les Ougaritains eux-mêmes.

Sous la forme d'une évocation poétique, ces textes « racontent » une cérémonie qui impliquait directement la famille royale. Par le soin mis à leur composition, ils se présentent comme de véritables œuvres littéraires, commémorant un moment fort
[24] Voir MARINATOS 1993 ; HUSSER 2008 ; MOUTON 2008 ; MOUTON \& PATRIER 2014.

[25] PARDEE 1993, p. 207.

[26] Cette conclusion va à l'encontre de l'opinion générale qui considère KTU 1.161 comme un livret rituel, en dépit de ses particularités formelles ici analysées, et du fait qu'il demeure difficile de préciser la réalité du rituel qu'il est censé décrire, « either the libretto of a funerary service for a king who recently died, or a ritual in commemoration of his death... »(RouILLARD 1999, p. 693). Voir aussi SURIANO 2009, p. 117. 
dans la vie de la maison royale d'Ugarit, en I'occurrence une cérémonie d'initiation de jeunes princes (encore non identifiés) et la sépulture du roi Niqmaddu IV [27]. Ils procèdent en mêlant au récitatif divers fragments du rituel de manière à en rappeler le déroulé, ainsi que des morceaux lyriques et mythologiques qui en soulignent la signification. Leur but n'était donc pas de guider I'accomplissement de ces rituels, mais de transmettre le souvenir de ces cérémonies jugées dignes de mémoire en raison de leur importance et de leur signification dans le contexte de l'idéologie royale. C'est pourquoi il nous faut considérer ce type d'écrits comme un genre littéraire spécifique et jusqu'ici mal identifié dans notre documentation [28], dont la fonction était davantage mémorielle et idéologique que proprement rituelle. Ils étaient engagés dans une célébration de la dynastie régnante et la construction de son prestige en rappelant son lien avec le monde divin à des moments clés de la vie : la sortie de la petite enfance (véritable naissance sociale) et la mort [29].

Considérés sous cet angle, ces textes soulèvent quelques questions subsidiaires, et tout d'abord celle de l'intérêt que représentait, pour les anciens Ougaritains - et plus précisément pour le milieu de la cour -, cette façon de célébrer la mémoire de ces cérémonies particulières. Disons rapidement que la qualité littéraire de ces textes en faisait des pièces agréables à lire et à entendre, faciles à mémoriser aussi, et qui devaient contribuer à entretenir une image idéalisée de la famille royale par le rappel de liturgies occasionnelles mais grandioses, à la manière des tableaux mettant en scène les souverains dans notre patrimoine européen (scènes de mariages, de couronnements, de visites aux armées, etc.).

Faut-il y voir des œuvres spontanées à l'initiative d'un poète de cour, ou des pièces de propagande répondant à un programme établi ? Il est difficile de répondre précisément à cette question, mais il faut se rappeler que le règne de Niqmaddu IV (ca 12201210) fut celui qui vit la composition et la rédaction, sous le stylet d'Ilimilku, des grandes œuvres mythologiques et épiques du corpus ougaritique, dont le Cycle de Baal (KTU 1.1 - 1.6) [30]. Il est inutile d'insister sur les liens étroits entretenus par cette littérature épique et l'idéologie royale, c'est un sujet qui a été abondamment étudié. Or, la composition de nos deux textes s'insère dans le même contexte idéologique et, selon toute vraisemblance, dans la même période de temps. La composition du Livret du banquet des ombres (KTU 1.161) peut être située aux alentours de 1210, au tout début de la régence de Sharelli (ca 1210-1200), puisqu'il évoque les funérailles de son époux. S'il est plus difficile d'être aussi précis pour le poème dit des « dieux gracieux » (KTU 1.23) et d'identifier les princes royaux qui en sont les protagonistes, sa proximité ici démontrée avec KTU 1.161 me paraît situer sa composition également dans ces dernières décennies de la vie du royaume. Cette période est marquée par une production littéraire abondante et de grande qualité, très impliquée dans les questions touchant à la royauté et dans la construction d'une identité dynastique, et nos deux textes y contribuaient de façon tout à fait originale.
[27] Sur un autre registre artistique, mais dans un contexte d'idéologie royale analogue, le relief du sarcophage d'Ahiram figurant certainement le repas funéraire du roi défunt, me semble présenter un bon équivalent iconographique au texte des funérailles de Niqmaddu. L'essentiel du rituel est représenté, non dans le détail de la matérialité des rites, mais dans l'imaginaire de leur signification ultime : le défunt reçoit I'hommage de sa cour à travers le banquet funéraire, comme il le faisait de son vivant. On y retrouve les mêmes objets symboliques de la fonction royale que dans KTU 1.161 : le trône, l'escabeau et la table. Pour une reprise récente des questions multiples posées par ce monument de l'archéologie phénicienne, voir REHM 2004.
[28] Certes, deux textes seulement documentent ce genre littéraire, mais la date tardive de leur composition (dans la chronologie du royaume d'Ugarit) peut suffire à expliquer cette rareté ainsi que leur focalisation sur la royauté, à l'instar de l'ensemble de la littérature ougaritique connue.

[29] Pour l'implication de ces textes dans l'idéologie royale, voir HUSSER 2017 pour KTU 1.23 et SURIANO 2009 pour KTU 1.161.

[30] De chacune de ces œuvres, il faut le souligner, nous ne connaissons qu'un seul exemplaire. La destruction de la ville intervint vers 1180 , de sorte que le temps a manqué, semble-t-il, pour faire de cette production littéraire, originale et de grande qualité, le socle d'une tradition scribale qui en aurait assuré la diffusion. 


\section{BIBLIOGRAPHIE}

Bordreurl, Pierre (dir.), 1991, Une bibliothèque au sud de la ville, Paris (RSO VII), p. 153-163.

Bordreuil, Pierre \& PARdee, Dennis, 1982, « Le rituel funéraire ougaritique RS 34.126», Syria 59, p. $121-128$.

Caquot, André, Tarragon, Jean-Michel de \& Cunchillos, Jesus-Luis, 1989, Textes ougaritiques. Tome 2 : Textes religieux, rituels, correspondance, Paris (LAPO 14).

De Moor, Johann C., 1987, An Anthology of Religious Texts from Ugarit, Leiden (Nisaba 16).

Del Olmo Lete, Gregorio, 1999, Canaanite Religion According to the Liturgical Texts of Ugarit, Bethesda.

FREU, Jacques, 2006, Histoire politique du royaume d'Ugarit, Paris.

Husser, Jean-Marie, 2008, « La mort d'Aqhat. Chasse et rites de passage à Ugarit », Revue de I'Histoire des Religions 225, p. 323-346.

HuSSER, Jean-Marie, 2017, « What if the goodly gods were bonnie princes? An integration rite for royal princes in KTU $1.23 »$, Ugarit Forschungen 48, p. 685-694.

LeVine, B. A. \& TARRAgon, Jean-Michel de, 1984, «Dead kings and rephaim. The patrons of the Ugaritic dynasty », Journal of the American Oriental Society 104, p. 649-659.

Marinatos, Nanno, 1993, Minoan Religion. Ritual, Image, and Symbol, chap. 10 : « Ritual contests, hunting, and rites of passage », Columbia S.C. (Studies in comparative religion), p. 201-220.

Mouton, Alice, 2008, Les Rituels de naissance kizzuwatniens. Un exemple de rite de passage en Anatolie hittite, Paris. Mouton, Alice \& PATrier, Julie (éd.), 2014, Life, Death and Coming of Age in Antiquity. Individual Rites of Passage in the Ancient Near East and Adjacent Regions, Leiden.

PARDee, Dennis, 1988, Les Textes para-mythologiques de la 64e campagne, Paris (RSO IV4).

Pardee, Dennis, 1993, « Poetry in Ugaritic Ritual Texts », in J. C. de Moor \& W. G. E. Watson (eds), Verse in Ancient Near Eastern Prose, Neukirchen-Vluyn (AOAT 42), p. 207-2018.

PARDee, Dennis, 2000, Les Textes rituels, 2 vol., Paris (RSO XII), vol. 2, p. 816-825.

PARDee, Dennis, 2002, Ritual and Cult at Ugarit, Atlanta (SBLWAW 10).

Reнm, Ellen, 2004, Dynastensarkophage mit szenischen Reliefs aus Byblos und Zypern. Teil 1.1 Der Ahiram-Sarkophag, Mainz-am-Rhein.

RouillaRD, Hedwige, 1999, s. v. Rephaim, dans K. van der Toorn, B. Becking \& P.W. van der Hor (éd.), Dictionary of Deities and Demons in the Bible, DDD, 2e édition, Leiden, Boston, p. 692-700.

SмIтн, Mark S., 2006, The Rituals and Myths of the Feast of the Goodly Gods of KTU/CAT 1.23, Atlanta (RBS 51).

SPRONK, Klaas, 1986, Beatific Afterlife in Ancient Israel and the Ancient Near East, Neukirchen-Vluyn (AOAT 219).

SURIANO, Matthew, 2009, «Dynasty building at Ugarit. The ritual and political context of KTU 1.161 », Aula Orientalis 27, p. $105-123$.

TsUMURA, David, 1993, « The interpretation of the Ugaritic funerary text KTU 1.161 », dans E. Matsushima (éd.), Official Cult and Popular Religion in the Ancient Near East, Heidelberg, p. 40-55.

VirolleaUd, Charles, 1933, «La naissance des dieux gracieux et beaux », Syria 14, p. 128-151. 